\title{
Correction to: Linear numerosity illusions in capuchin monkeys (Sapajus apella), rhesus macaques (Macaca mulatta), and humans (Homo sapiens)
}

\author{
Audrey E. Parrish ${ }^{1}$ Michael J. Beran ${ }^{2,3} \cdot$ Christian Agrillo $^{4}$
}

Published online: 19 July 2019

๑) Springer-Verlag GmbH Germany, part of Springer Nature 2019

\section{Correction to: Animal Cognition}

https://doi.org/10.1007/s10071-019-01288-9

In the original publication, values of last three rows in Tables 1 and 2 were incorrectly published. The values are corrected and the complete Tables 1 and 2 are given below.

The original article can be found online at https://doi.org/10.1007/ s10071-019-01288-9.

Audrey E. Parrish audrey.parrish1@gmail.com

$\triangle$ Christian Agrillo christian.agrillo@unipd.it

1 Department of Psychology, The Citadel, 171 Moultrie St, Charleston, SC 29409, USA

2 Language Research Center, Georgia State University, Atlanta, GA, USA

3 Department of Psychology, Georgia State University, Atlanta, GA, USA

4 Department of General Psychology, University of Padova, Via Venezia 8, 35131 Padua, Italy 


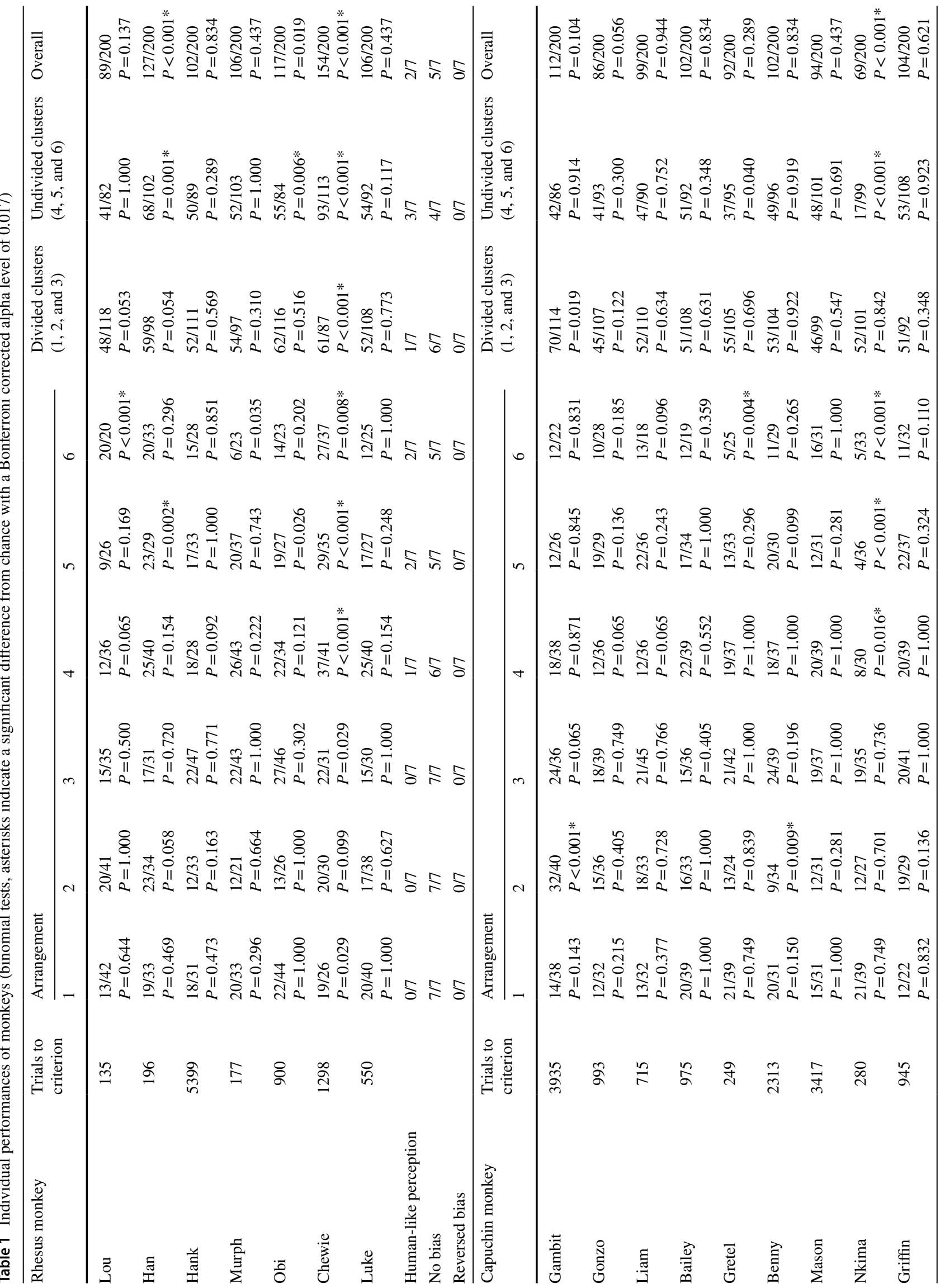




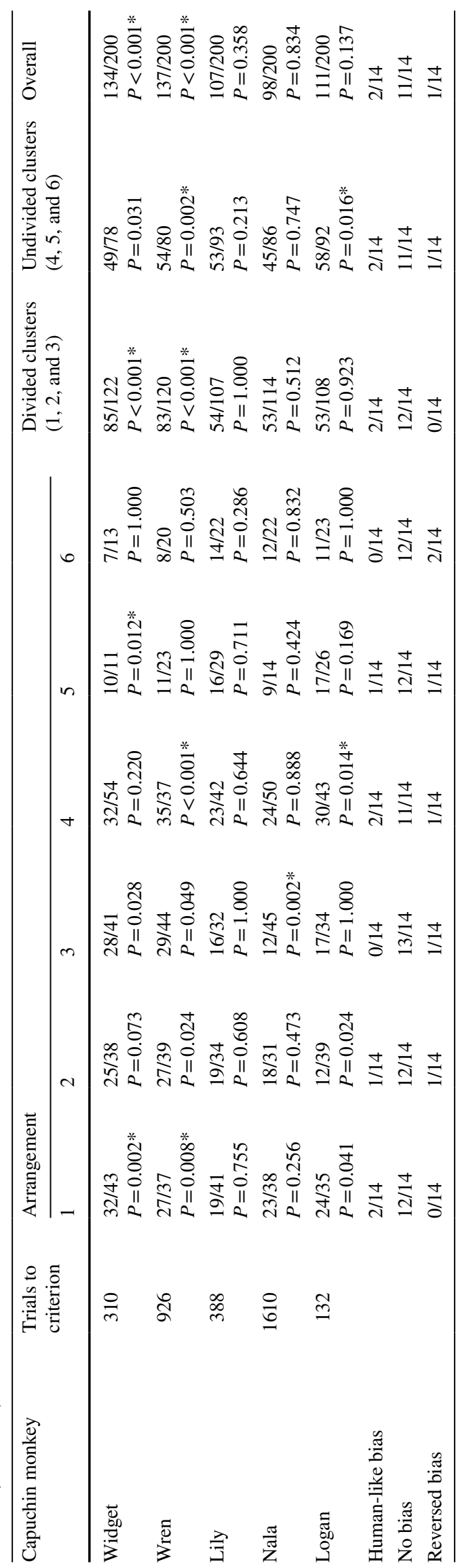


Table 2 Human data (binomial tests, asterisks indicate a significant difference from chance with a Bonferroni corrected alpha level of .017)

\begin{tabular}{|c|c|c|c|c|c|c|c|c|c|c|}
\hline \multirow[t]{2}{*}{ Subject } & \multirow{2}{*}{$\begin{array}{l}\text { Trials to } \\
\text { criterion }\end{array}$} & \multicolumn{6}{|c|}{ Arrangement } & \multirow{2}{*}{$\begin{array}{l}\text { Divided } \\
\text { clusters } \\
(1,2,3)\end{array}$} & \multirow{2}{*}{$\begin{array}{l}\text { Undivided } \\
\text { clusters } \\
(4,5,6)\end{array}$} & \multirow[t]{2}{*}{ Overall } \\
\hline & & 1 & 2 & 3 & 4 & 5 & 6 & & & \\
\hline 1 & 21 & $\begin{array}{l}13 / 13 \\
P<0.001 *\end{array}$ & $\begin{array}{l}15 / 15 \\
P<0.001 *\end{array}$ & $\begin{array}{l}22 / 23 \\
P<0.001 *\end{array}$ & $\begin{array}{l}6 / 16 \\
P=0.454\end{array}$ & $\begin{array}{l}9 / 18 \\
P=1.000\end{array}$ & $\begin{array}{l}6 / 15 \\
P=0.607\end{array}$ & $\begin{array}{l}50 / 51 \\
P<0.001 *\end{array}$ & $\begin{array}{l}21 / 46 \\
P=0.659\end{array}$ & $\begin{array}{l}71 / 100 \\
P<0.001 *\end{array}$ \\
\hline 2 & 21 & $\begin{array}{l}12 / 13 \\
P=0.003\end{array}$ & $\begin{array}{l}22 / 23 \\
P<0.001 *\end{array}$ & $\begin{array}{l}17 / 17 \\
P<0.001 *\end{array}$ & $\begin{array}{l}16 / 22 \\
P=0.052\end{array}$ & $\begin{array}{l}13 / 17 \\
P=0.049\end{array}$ & $\begin{array}{l}4 / 8 \\
P=1.000\end{array}$ & $\begin{array}{l}51 / 52 \\
P<0.001^{*}\end{array}$ & $\begin{array}{l}33 / 47 \\
P=0.008^{*}\end{array}$ & $\begin{array}{l}84 / 100 \\
P<0.001 *\end{array}$ \\
\hline 3 & 21 & $\begin{array}{l}11 / 14 \\
P=0.057\end{array}$ & $\begin{array}{l}17 / 21 \\
P=0.007\end{array}$ & $\begin{array}{l}10 / 20 \\
P=1.000\end{array}$ & $\begin{array}{l}12 / 21 \\
P=0.664\end{array}$ & $\begin{array}{l}9 / 13 \\
P=0.267\end{array}$ & $\begin{array}{l}7 / 11 \\
P=0.549\end{array}$ & $\begin{array}{l}38 / 55 \\
P=0.006^{*}\end{array}$ & $\begin{array}{l}28 / 45 \\
P=0.135\end{array}$ & $\begin{array}{l}66 / 100 \\
P=0.002^{*}\end{array}$ \\
\hline 4 & 26 & $\begin{array}{l}21 / 23 \\
P<0.001 *\end{array}$ & $\begin{array}{l}10 / 10 \\
P=0.002\end{array}$ & $\begin{array}{l}13 / 19 \\
P=0.167\end{array}$ & $\begin{array}{l}10 / 23 \\
P=0.678\end{array}$ & $\begin{array}{l}3 / 12 \\
P=0.146\end{array}$ & $\begin{array}{l}6 / 13 \\
P=1.000\end{array}$ & $\begin{array}{l}44 / 52 \\
P<0.001 *\end{array}$ & $\begin{array}{l}19 / 48 \\
P=0.193\end{array}$ & $\begin{array}{l}63 / 100 \\
P=0.012^{*}\end{array}$ \\
\hline 5 & 21 & $\begin{array}{l}19 / 19 \\
P<0.001 *\end{array}$ & $\begin{array}{l}19 / 19 \\
P<0.001 *\end{array}$ & $\begin{array}{l}21 / 21 \\
P<0.001 *\end{array}$ & $\begin{array}{l}9 / 14 \\
P=0.424\end{array}$ & $\begin{array}{l}7 / 14 \\
P=0.100\end{array}$ & $\begin{array}{l}5 / 13 \\
P=0.581\end{array}$ & $\begin{array}{l}59 / 59 \\
P<0.001 *\end{array}$ & $\begin{array}{l}21 / 41 \\
P=1.000\end{array}$ & $\begin{array}{l}80 / 100 \\
P<0.001 *\end{array}$ \\
\hline 6 & 37 & $\begin{array}{l}13 / 13 \\
P<0.001 *\end{array}$ & $\begin{array}{l}14 / 16 \\
P=0.004 *\end{array}$ & $\begin{array}{l}13 / 19 \\
P=0.167\end{array}$ & $\begin{array}{l}8 / 21 \\
P=0.383\end{array}$ & $\begin{array}{l}11 / 18 \\
P=0.481\end{array}$ & $\begin{array}{l}2 / 13 \\
P=0.022\end{array}$ & $\begin{array}{l}40 / 48 \\
P<0.001^{*}\end{array}$ & $\begin{array}{l}21 / 52 \\
P=0.212\end{array}$ & $\begin{array}{l}61 / 100 \\
P=0.035\end{array}$ \\
\hline 7 & 24 & $\begin{array}{l}12 / 12 \\
P<0.001 *\end{array}$ & $\begin{array}{l}18 / 18 \\
P<0.001 *\end{array}$ & $\begin{array}{l}18 / 21 \\
P=0.001\end{array}$ & $\begin{array}{l}11 / 20 \\
P=0.824\end{array}$ & $\begin{array}{l}6 / 12 \\
P=1.000\end{array}$ & $\begin{array}{l}9 / 17 \\
P=1.000\end{array}$ & $\begin{array}{l}48 / 51 \\
P<0.001^{*}\end{array}$ & $\begin{array}{l}26 / 49 \\
P=0.775\end{array}$ & $\begin{array}{l}74 / 100 \\
P<0.001^{*}\end{array}$ \\
\hline 8 & 21 & $\begin{array}{l}16 / 17 \\
P<0.001 *\end{array}$ & $\begin{array}{l}20 / 22 \\
P<0.001 *\end{array}$ & $\begin{array}{l}14 / 14 \\
P<0.001 *\end{array}$ & $\begin{array}{l}10 / 17 \\
P=0.629\end{array}$ & $\begin{array}{l}11 / 19 \\
P=0.648\end{array}$ & $\begin{array}{l}4 / 11 \\
P=0.549\end{array}$ & $\begin{array}{l}50 / 53 \\
P<0.001^{*}\end{array}$ & $\begin{array}{l}25 / 47 \\
P=0.771\end{array}$ & $\begin{array}{l}75 / 100 \\
P<0.001^{*}\end{array}$ \\
\hline 9 & 21 & $\begin{array}{l}16 / 16 \\
P<0.001 *\end{array}$ & $\begin{array}{l}17 / 17 \\
P<0.001 *\end{array}$ & $\begin{array}{l}21 / 21 \\
P<0.001 *\end{array}$ & $\begin{array}{l}7 / 13 \\
P=1.000\end{array}$ & $\begin{array}{l}15 / 18 \\
P=0.008 *\end{array}$ & $\begin{array}{l}7 / 15 \\
P=1.000\end{array}$ & $\begin{array}{l}54 / 54 \\
P<0.001 *\end{array}$ & $\begin{array}{l}29 / 46 \\
P=0.104\end{array}$ & $\begin{array}{l}83 / 100 \\
P<0.001^{*}\end{array}$ \\
\hline 10 & 21 & $\begin{array}{l}15 / 15 \\
P<0.001 *\end{array}$ & $\begin{array}{l}18 / 18 \\
P<0.001 *\end{array}$ & $\begin{array}{l}14 / 19 \\
P=0.064\end{array}$ & $\begin{array}{l}7 / 18 \\
P=0.481\end{array}$ & $\begin{array}{l}10 / 13 \\
P=0.092\end{array}$ & $\begin{array}{l}6 / 17 \\
P=0.332\end{array}$ & $\begin{array}{l}47 / 52 \\
P<0.001 *\end{array}$ & $\begin{array}{l}23 / 48 \\
P=0.885\end{array}$ & $\begin{array}{l}70 / 100 \\
P<0.001 *\end{array}$ \\
\hline 11 & 22 & $\begin{array}{l}10 / 12 \\
P=0.039\end{array}$ & $\begin{array}{l}14 / 15 \\
P=0.001\end{array}$ & $\begin{array}{l}8 / 27 \\
P=0.052\end{array}$ & $\begin{array}{l}13 / 14 \\
P=0.002 *\end{array}$ & $\begin{array}{l}2 / 14 \\
P=0.013 *\end{array}$ & $\begin{array}{l}3 / 18 \\
P=0.008 *\end{array}$ & $\begin{array}{l}32 / 54 \\
P=0.220\end{array}$ & $\begin{array}{l}18 / 46 \\
P=0.184\end{array}$ & $\begin{array}{l}50 / 100 \\
P=1.000\end{array}$ \\
\hline 12 & 21 & $\begin{array}{l}18 / 18 \\
P<0.001 *\end{array}$ & $\begin{array}{l}30 / 30 \\
P<0.001 *\end{array}$ & $\begin{array}{l}18 / 18 \\
P<0.001 *\end{array}$ & $\begin{array}{l}5 / 10 \\
P=1.000\end{array}$ & $\begin{array}{l}12 / 12 \\
P<0.001 *\end{array}$ & $\begin{array}{l}9 / 12 \\
P=0.146\end{array}$ & $\begin{array}{l}66 / 66 \\
P<0.001^{*}\end{array}$ & $\begin{array}{l}26 / 34 \\
P=0.003^{*}\end{array}$ & $\begin{array}{l}92 / 100 \\
P<0.001^{*}\end{array}$ \\
\hline 13 & 20 & $\begin{array}{l}16 / 19 \\
P=0.004 *\end{array}$ & $\begin{array}{l}11 / 19 \\
P=0.648\end{array}$ & $\begin{array}{l}9 / 16 \\
P=0.804\end{array}$ & $\begin{array}{l}16 / 16 \\
P<0.001 *\end{array}$ & $\begin{array}{l}5 / 16 \\
P=0.210\end{array}$ & $\begin{array}{l}6 / 14 \\
P=0.791\end{array}$ & $\begin{array}{l}36 / 54 \\
P=0.020\end{array}$ & $\begin{array}{l}27 / 46 \\
P=0.302\end{array}$ & $\begin{array}{l}63 / 100 \\
P=0.012^{*}\end{array}$ \\
\hline 14 & 21 & $\begin{array}{l}16 / 16 \\
P<0.001 *\end{array}$ & $\begin{array}{l}32 / 32 \\
P<0.001 *\end{array}$ & $\begin{array}{l}14 / 14 \\
P<0.001 *\end{array}$ & $\begin{array}{l}4 / 8 \\
P=1.000\end{array}$ & $\begin{array}{l}16 / 16 \\
P<0.001 *\end{array}$ & $\begin{array}{l}12 / 14 \\
P=0.013\end{array}$ & $\begin{array}{l}62 / 62 \\
P<0.001 *\end{array}$ & $\begin{array}{l}32 / 38 \\
P<0.001^{*}\end{array}$ & $\begin{array}{l}94 / 100 \\
P<0.001^{*}\end{array}$ \\
\hline 15 & 22 & $\begin{array}{l}18 / 22 \\
P=0.004 *\end{array}$ & $\begin{array}{l}9 / 9 \\
P=0.004^{*}\end{array}$ & $\begin{array}{l}15 / 15 \\
P<0.001 *\end{array}$ & $\begin{array}{l}17 / 18 \\
P<0.001 *\end{array}$ & $\begin{array}{l}3 / 18 \\
P=0.021\end{array}$ & $\begin{array}{l}3 / 18 \\
P=0.008^{*}\end{array}$ & $\begin{array}{l}42 / 46 \\
P<0.001^{*}\end{array}$ & $\begin{array}{l}23 / 54 \\
P=0.341\end{array}$ & $\begin{array}{l}65 / 100 \\
P=0.004^{*}\end{array}$ \\
\hline 16 & 24 & $\begin{array}{l}19 / 19 \\
P<0.001 *\end{array}$ & $\begin{array}{l}16 / 18 \\
P=0.001\end{array}$ & $\begin{array}{l}18 / 19 \\
P<0.001 *\end{array}$ & $\begin{array}{l}17 / 17 \\
P<0.001 *\end{array}$ & $\begin{array}{l}12 / 12 \\
P<0.001 *\end{array}$ & $\begin{array}{l}14 / 15 \\
P<0.001 *\end{array}$ & $\begin{array}{l}53 / 56 \\
P<0.001^{*}\end{array}$ & $\begin{array}{l}43 / 44 \\
P<0.001^{*}\end{array}$ & $\begin{array}{l}96 / 100 \\
P<0.001^{*}\end{array}$ \\
\hline 17 & 22 & $\begin{array}{l}17 / 18 \\
P<0.001 *\end{array}$ & $\begin{array}{l}19 / 21 \\
P<0.001 *\end{array}$ & $\begin{array}{l}12 / 21 \\
P=0.664\end{array}$ & $\begin{array}{l}11 / 18 \\
P=0.481\end{array}$ & $\begin{array}{l}13 / 14 \\
P=0.002\end{array}$ & $\begin{array}{l}6 / 8 \\
P=0.289\end{array}$ & $\begin{array}{l}48 / 60 \\
P<0.001^{*}\end{array}$ & $\begin{array}{l}30 / 40 \\
P=0.002^{*}\end{array}$ & $\begin{array}{l}78 / 100 \\
P<0.001^{*}\end{array}$ \\
\hline 18 & 21 & $\begin{array}{l}13 / 13 \\
P<0.001 *\end{array}$ & $\begin{array}{l}19 / 19 \\
P<0.001 *\end{array}$ & $\begin{array}{l}17 / 17 \\
P<0.001 *\end{array}$ & $\begin{array}{l}15 / 21 \\
P=0.078\end{array}$ & $\begin{array}{l}11 / 12 \\
P=0.006^{*}\end{array}$ & $\begin{array}{l}13 / 18 \\
P=0.096\end{array}$ & $\begin{array}{l}49 / 49 \\
P<0.001 *\end{array}$ & $\begin{array}{l}39 / 51 \\
P<0.001 *\end{array}$ & $\begin{array}{l}88 / 100 \\
P<0.001^{*}\end{array}$ \\
\hline 19 & 21 & $\begin{array}{l}19 / 20 \\
P<0.001 *\end{array}$ & $\begin{array}{l}20 / 21 \\
P<0.001 *\end{array}$ & $\begin{array}{l}11 / 15 \\
P=0.118\end{array}$ & $\begin{array}{l}18 / 20 \\
P<0.001 *\end{array}$ & $\begin{array}{l}12 / 15 \\
P=0.035\end{array}$ & $\begin{array}{l}4 / 9 \\
P=1.000\end{array}$ & $\begin{array}{l}50 / 56 \\
P<0.001 *\end{array}$ & $\begin{array}{l}34 / 44 \\
P<0.001^{*}\end{array}$ & $\begin{array}{l}84 / 100 \\
P<0.001^{*}\end{array}$ \\
\hline 20 & 20 & $\begin{array}{l}19 / 19 \\
P<0.001 *\end{array}$ & $\begin{array}{l}17 / 17 \\
P<0.001 *\end{array}$ & $\begin{array}{l}22 / 22 \\
P<0.001 *\end{array}$ & $\begin{array}{l}10 / 23 \\
P=0.678\end{array}$ & $\begin{array}{l}11 / 11 \\
P<0.001 *\end{array}$ & $\begin{array}{l}8 / 8 \\
P=0.008^{*}\end{array}$ & $\begin{array}{l}58 / 58 \\
P<0.001 *\end{array}$ & $\begin{array}{l}29 / 42 \\
P=0.020\end{array}$ & $\begin{array}{l}87 / 100 \\
P<0.001^{*}\end{array}$ \\
\hline Illusory bias & & $18 / 20$ & $19 / 20$ & $12 / 20$ & $5 / 20$ & $7 / 20$ & $3 / 20$ & $18 / 20$ & $7 / 20$ & $18 / 20$ \\
\hline No bias & & $2 / 20$ & $1 / 20$ & $8 / 20$ & $15 / 20$ & $12 / 20$ & $15 / 20$ & $2 / 20$ & $13 / 20$ & $2 / 20$ \\
\hline Reversed bias & & $0 / 20$ & $0 / 20$ & $0 / 20$ & $0 / 20$ & $1 / 20$ & $2 / 20$ & $0 / 20$ & $0 / 20$ & $0 / 20$ \\
\hline
\end{tabular}

